\title{
Comparing Treatment Approaches on Health Outcomes and Quality of Life for People Living with HIV Infection in Kasarani Kenya
}

\author{
By Teresia Mutiso* \\ Mary Ann Swain ${ }^{+}$
}

\begin{abstract}
Two existing clinical models were studied for their relative effectiveness in promoting positive perceived quality of life and in managing the course of HIV. One treatment approach was Peer Social Support (PSS) used primarily in low social economic neighborhoods with limited access to highly organized medical care. The second approach, Comprehensive Clinic Care (CCC), was based more in a medical model. Patients went to an urban medical facility for regular follow-up appointments where medical examinations and patient education were provided. The objective of this study was to determine if there were differences in treatment outcomes between these two clinical models. The study employed a convenience sample of 103 subjects for the Peer Social Support group and 90 for the Comprehensive Clinic Care group. Retrospective time's series design was used. Statistical measures used were; Univariate analysis of variance, chi square and one way repeated measures of ANOVA. The CCC groups had higher mean physical component scores for quality of life, $X=52.5$ (SD 6.8), than those in the PSS groups, $X=49.5$ (SD 7.5), $F(1,191)=8.4, p=0.006$. The mean mental component quality of life score was also higher for the CCC group X=51.4, (SD 9.3) than the PSS group, X=47.7, $(S D$ 9.1), $F(1,191)=7.8, p=0.006$. Weight showed a similar pattern, being higher for CCC, $X=68(S D$ 13.1) than PSS group $X=63.5$ (SD 12.5), $t(182)=-2.36, p=0.02$. The PSS group did have a higher CD4 count, $X=468$ (SD 200), than the CCC group, $X=356$ (SD 281), $F(1,162)=8.89, p=0.003$. No difference was observed in retention, PSS subjects returned for $90 \%$ of their appointments, and the CCC group for $87 \%$ of their appointments, $X^{2}(3$, $N=181)=4.39, p=0.222$. Both models appear to work for the clientele they serve. CCC is a good model for urban areas. PSS is a less expensive clinic model that is shown here to work in rural areas. Since nurses manage these rural clinics, more nurses need to be prepared to provide in low income resource settings.
\end{abstract}

Keywords: Health outcomes, HIV/AIDS, Quality of Life

\section{Introduction}

HIV/AIDS continues to be a major public health concern globally, particularly in Sub-Saharan Africa. The emergence of Antiretroviral Therapy (ART) has enabled developed countries to significantly reduce the number of deaths associated with HIV infection and to change HIV from an acutely deadly disease into a chronic condition that is accompanied by a long life (Lewden et al. 2007). But Sub-Saharan Africa has encountered delays in roll out of ARTs due to the substantial financial and human resources needed to establish and sustain an HIV care infrastructure. Two-thirds of people diagnosed with HIV live in Sub-Saharan Africa, but only 3\% of world health care personnel work there, and only $1 \%$ of world health expenditures go there

\footnotetext{
* PhD Student, Binghamton University, USA.

${ }^{\dagger}$ Director of the PhD Program DSON, Binghamton University, USA.
} 
(WHO 2006). Ways must be found to address this substantial health care problem.

But addressing the availability of ARTs is not the only challenge. Although Arts have changed the life course of HIV infection from an acutely fatal disease to a life long illness, there are still challenges affecting its success, particularly in resource-limited settings. These challenges include life long duration of therapy; serious liver, kidney, and other organ toxicities of long term therapy; the emergence of drug resistance and rapid viral replication; the possibility of re-infection among the HIV patients with different HIV strains; and issues of alcohol, and drug abuse associated with risk behaviors and nonadherence (Broder 2010)

Kenya currently has 1.2 million people living with HIV as a chronic infection. HIV prevalence among adults aged 15 to 64 years was estimated at $5.6 \%$ in 2012. The number of people living with HIV has remained steady after the introduction of anti-retroviral therapy. Fifty-eight percent of HIV-infected persons aged 15-64 years were eligible for antiretroviral therapy (ART) treatment for HIV infection based on a CD4+ T-cell count of 350 cells $/ \mathrm{ml}$. Between them, 63\% were currently on ART. However, viral suppression among all HIV-infected persons, regardless of awareness of HIV status and ART use was low, at $40 \%$ of all the infected persons. Attrition and lack of adherence are the two major causes of treatment failure, development of drug resistance, HIV disease progression, and death (Fox and Rosen 2010, PopEleches et al. 2011).

Unge et al. (2010) note that living below the poverty level; low education and lack of support (not having a formal treatment buddy) are significant risk factors for adherence. Providing care to HIV infected persons who need it throughout Kenya is hampered in low income and resource-limited communities by poor infrastructure (poor health care delivery system, poor public health monitoring and regulation); long distances to access health care services, and lack of personnel to man the available health centers. These deficiencies call for a variety of ways to help persons living with HIV infection to deal with the disease process.

\section{Problem Statement}

Living with chronic HIV infections has many negative effects on an individual's physical, emotional and social life. These changes in overall quality of life probably affect patients' adherence to treatment regimens. Nurses by their holistic approach in care, are well prepared for helping these individuals. But nurses lack clarity as to how to ensure that those infected with HIV take full advantage of treatments known to have a positive effect. The question becomes "How do nurses encourage those with HIV chronic infections on ART to seek regular follow-up care and motivate them to follow a treatment regimen for life?". 


\section{Purpose}

Treatment methods are often initiated because they are thought to be "a good idea". But often the methods are not evaluated for their effectiveness. In Kenya there are different clinical models for assisting individuals manage their HIV conditions. Two of these are Comprehensive Clinic Care and Peer Social Support. The purpose of this study was to evaluate the relative effectiveness of these two existing treatment approaches to provide guidance to nurses and public health officials in the design of care for individuals with HIV infections.

\section{Methodology}

\section{Clinical Models}

\section{Comprehensive clinic care model}

Comprehensive Clinic Care is delivered in an urban medical facility. Patients come to the clinic from the outskirts of Nairobi city. Once patients have been diagnosed with HIV they receive a comprehensive medical assessment, anti-retroviral therapy depending on their CD4 count, and patient education (including the importance of medication adherence and counseling on ways to care for themselves based on their individual needs). Patients are scheduled for follow-up appointments monthly.

\section{Peer social support model}

Medical treatment for those in Peer Social Support occurs in a variety of public and private clinics throughout the outskirts of Nairobi. After individuals are diagnosed with an HIV infection, further assessment is done to establish their needs. Nurses in those clinics refer individuals to PSS who are debilitated, with little or no social support, and/or are unemployed. In addition, village community health workers also do door to door visitations and refer HIV individuals who have an unstable social network to PSS. The PSS groups were all initiated and facilitated by nurses. There are four PSS groups in different neighborhoods in the slum areas of Nairobi. When groups meet they are encouraged to share their experiences (both positive and negative) about the disease in order to teach each other ways to live more fruitfully. They offer each other comfort and encouragement when feelings of discouragement and hopelessness arise. They reinforce the need for and benefit of follow-up treatments.

\section{Selection and Recruitment}

All participants had to have been in treatment for at least the last 18 months after diagnosis and have the ability to read and speak English. For both clinic models the participants represent a convenience sample of those who met both the inclusion criteria and were willing to participate. For the PSS group 
recruitment consisted of posting fryers in a community center asking individuals who were interested to contact the director, who explained the study and their role in it. CCC participants were recruited personally by employees in the clinic as they came for their monthly appointments. All individuals who expressed interest were informed of the purpose of the study, asked to sign a consent form explaining their role in the project and that they could leave a session or the study at any time without affecting their future care

\section{Data Collected}

The following demographic data were collected to compare the membership in the two groups: age, gender, occupation, education, and year of diagnosis. To compare the effectiveness of the two clinical models weight, CD4 counts, survey responses about subjects' quality of life, and the number of missed appointments were collected to ascertain the relative effectiveness of the different models.

\section{Quality of life survey}

The SF-12v2 survey measures eight domains of health related quality of life (physical function, social function, physical role limitation, emotional role limitation, vitality, pain, mental health and general health). The results of the eight domains are aggregated into two summary scores, physical component scores and mental component scores (PCS \& MCS) respectively. According to Luo et al. (2003) Cronbach's alpha for the physical component score for the SF-12 was 0.77; Cronbach's alpha for the mental component was 0.8 - both exceeding the recommended level of 0.70 . Concurrent validity for SF-12 was established by comparing back pain and the degree of overall well-being. Back pain was significantly, and negatively correlated with the physical component score $-12(\mathrm{R}=-0.405, \mathrm{p}<0.0001)$ and mental component score $-12(\mathrm{R}=-0.326$, $\mathrm{p}<0.0001)$.

\section{Study design}

A retrospective time series design was used. After signing the consent form all data described above were collected at the time of enrollment. Medical records were then examined at the date of initial visit, 6, 12, and 18 months prior to entry into the study to extract information from previous clinic visits.

\section{Research Questions}

Is Comprehensive Clinic Care or Peer Social Support more effective in fostering health outcomes for persons with HIV? More specifically, are there differences in weight, CD4 counts, and physical or psychological health status between those being seen in CCC or those who are in PSS groups? Are there differences in persistence in seeking medical care between the two groups? 


\section{Results}

\section{Demographic Data}

As can be seen in Table 1, the two groups differed in their composition. Participants in the Peer Social Support groups were more likely to be female, with less education, not employed, and without a partner. In other words, they had fewer resources upon which to draw. Viewing employment and having a partner as being connected to others presumably means that members of the PSS group had less social support in their daily lives than members of the CCC group.

Table 1. Demographic Comparisons for Clinical Models

\begin{tabular}{|c|c|c|c|c|c|}
\hline \multicolumn{1}{|c|}{ Variable } & PSS & CCC & Statistic & Probability & DF \\
\hline Age & & & & & \\
\hline Gender & 41 & 40 & $\mathrm{t}=0.93$ & $p=0.4$ & 191 \\
\hline & & & & & \\
\hline Female & & & $X^{2}=6.02$ & $p=0.014$ & 1 \\
\hline Male & $87(87 \%)$ & $62(69 \%)$ & & & \\
\hline Employment & $16(13 \%)$ & $27(31 \%)$ & & & \\
\hline & & & & & 1 \\
\hline Employed & & & $X^{2}=19.99$ & $p=0.000$ & 1 \\
\hline Unemployed & $47(47 \%)$ & $69(79 \%)$ & & & \\
\hline Relationship & $52(52 \%)$ & $18(21 \%)$ & & & \\
\hline & & & & & \\
\hline Yes & & & $X^{2}=11.24$ & $p=0.001$ & 1 \\
\hline No & $36(35 \%)$ & $53(60 \%)$ & & & \\
\hline Education & $66(65 \%)$ & $36(40 \%)$ & & & \\
\hline & & & & & \\
\hline$>$ Secondary School & $15(15 \%)$ & $30(64 \%)$ & & & \\
\hline Secondary School & $29(28 \%)$ & $16(34 \%)$ & & & \\
\hline Primary School & $59(58 \%)$ & $1(2 \%)$ & & & \\
\hline Year Diagnosed & & & & & \\
\hline & 2007 & 2009 & $\mathrm{t}=5.4$ & $p=0.000$ & 186 \\
\hline Source Authors calcula & & & & \\
\hline
\end{tabular}

Source: Authors calculations.

This difference was at least partly a result of the fact that nurses referred patients without a support system to the PSS group. The differences in education and employment are not surprising given that the CCC group was drawn from higher social economic strata. Finally, members of the PSS group had, on average, been dealing with an HIV diagnosis for a longer period of time. On the one hand this means they had longer exposure to ARTs. On the other hand this means they probably also experienced more untoward sequelae of the disease. 


\section{Findings Related to Research Questions}

Quality of life

The CCC group had higher physical component mean scores 52.5 $(\mathrm{SD}=6.8)$, than the PSS group $49.5(\mathrm{SD}=7.5)$. This difference was statistically significant $\mathrm{F}(1,191)=8.4, \mathrm{p}=0.004$, partial eta squared 0.42 . The mental component summary scores results were also significantly different between the two groups $\mathrm{F}(1,191)=7.8, \mathrm{p}=0.006$. The partial eta squared was 0.039 . The $\mathrm{CCC}$ group had a mean mental component summary score of $51.4(\mathrm{SD}=9.3)$, compared to the PSS group whose mean was $47.7(\mathrm{SD}=9.1)$.

Weight and weight gain

At the entry into the study the mean weight for the CCC group was higher, 68 ( $\mathrm{SD}=13.1)$, compared to the PSS group, 63.5 ( $\mathrm{SD}=12.5)$. The findings were statistically significant $\mathrm{F}(1,180)=5.9, \mathrm{p}=0.02, \mathrm{n}=0.032$. The results for a repeated measures ANOVA indicated no significant difference between the groups in weight change over time, Wilks $\mathrm{A}=0.649 \mathrm{~F}(3,67)=1.20, \mathrm{p}=0.32$, $\mathrm{n}^{2}=0.05$. But it was interesting to note that there was a statistically significant difference within each group reflecting weight gains overtime. On average the PSS group gained $8.5 \mathrm{~kg}(18.7 \mathrm{lbs}$.) and the CCC group gained $5.1 \mathrm{~kg}(11.22$ lbs.) between weights recorded 18 months prior to entry into the study and weights recorded at entry, $A=0.610 F(3,67)=10.0, p=0.000, n^{2}=0.31$

\section{CD4 count}

Comparisons for the CD4 count at entry to the project revealed that subjects belonging to the Peer Social Support group had a mean CD4 count of 468 ( $\mathrm{SD}=200$ ) while the comprehensive Clinic care group had a mean CD4 count of $356(\mathrm{SD}=281)$. This was a statistically significant difference, $\mathrm{F}$ $(1,162)=8.89, \mathrm{p}=0.003, \mathrm{n}^{2}=0.52$.

The original design for the study included retrospective reviews of medical records to collect data on CD4 counts overtime. Unfortunately, these data were largely missing in the subjects' medical records. Of the 91 subjects who had a CD4 count at entry into the project, only 25 subjects had recorded counts 6 months back, 19 a year back, and 14 a year and a half back. Thus it was not possible to determine if there were any differences between the two groups in their CD4 counts over time.

\section{Missed Appointments}

Peer Social Support had a mean of $3.2(\mathrm{SD}=8.7)$ missed appointments, while Comprehensive Care Group had 3.1 ( $\mathrm{SD}=9.1$ ). The PSS group kept 90\% of their appointments and the CCC group kept $87 \%$ of their appointments. There was no difference between the two treatment groups, $\mathrm{X}^{2}(3, \mathrm{~N}=181)$ $=4.39, p=0.222$. 


\section{Discussion}

Individuals being treated within these two clinical models differ in gender, marital status, whether or not they are employed, and the duration of living with the disease. At the time of the initial data collection, these differences confirm the referral pattern for the PSS group who were judged to be worse off in the perspective of social connectedness. Having more participants gainfully employed with more financial resources may account for why the CCC group had significantly higher means for 3 outcomes: physical quality of life, mental quality of life, and weight. If an earlier diagnosis meant earlier exposure to ART, the fact that the PSS group had significantly higher CD4 counts than those in CCC group is understandable. However, the discrepancy between subjective quality of life findings and objective CD4 counts warrants further exploration. If subjects are healthier with respect to the disease, why do they not have an accompanying higher perceived quality of life? Perhaps all these differences reflect the fact that PSS subjects were living in poverty and social isolation.

Although the CCC group appeared to have an overall advantage in terms of the outcomes achieved, both nursing approaches (CCC and PSS) resulted in comparatively better health outcomes. Other Studies carried out in South Africa, and Malawi had similar findings indicating that the Social support network was essential for those living with HIV/AIDS (Kabore et al. 2010, Nachega et al. 2006). For example, mean scores for the physical and mental quality of life scores for the CCC group were 52.5 and 51.4, while for the PSS group they were 49.5 and 47.7. These means for both groups were better than scores reported by Ion et al. (2011) at 47.7 and 44.0 respectively and by Visiwanatha et al. (2005), which were $(41.0,41.9)$ respectively. The mean CD4 count for the PSS group was significantly higher at 468 cells $/ \mathrm{ml}$ than that of the CCC group at 356 cells $/ \mathrm{ml}$. But clinically, both the PSS and CCC groups were doing better than other clinical benchmarks such as Bekker et al. (2006) with 110 cells/ $\mu$ l or Kaplan et al. (2003) who considered 350 cells $/ \mathrm{ml}$ the cut point; those who were below were considered as having a low CD4 count, and those above 350 cells $/ \mathrm{ml}$ were considered as having a high CD4 count. These two clinical models appeared to meet the needs of patients, as indicated by the relative high rates of using followup care in both groups. This was comparable to those found in Bekker et al. (2006) at $91 \%$ and higher than a meta-analysis study on published results including 32 scientific reports from 13 countries in sub-Saharan Africa which revealed that the average retention rate at 12 months was 75\% (Rosen and Fox 2011).

\section{Limitations}

This was a convenience sampling. Thus, it was fast and easy to collect data, subjects were readily available and the study was cost effective. But any study that is not randomized introduces some decree of bias. Use of already existing groups, like those in this study, though permissible; make group comparisons more problematic (Campbell et al. 1963). As can be the case when selection is not random, demographic characteristics varied between the two groups. Secondly, the monitoring of treatment outcomes was inconsistent. It is not possible to tell how well either the clinical model would have fared, if 
CD4 counts had been taken and used to manage treatment over time. Finally, the quality of life measures were self-reports. Bias can enter if individuals want to provide a socially acceptable answer. Perhaps because of the perceived prestige of the Comprehensive Care Clinic and the community stature of those it serves, its participants were more likely to report they were doing better.

\section{Conclusions and Recommendations}

\section{Conclusion}

The purpose of this study was to determine if there were differences in treatment outcomes for two different clinical models. While there are differences between the two treatment approaches, the differences are not substantial enough to warrant recommending one clinical model over the other. Both treatment approaches resulted in health related outcomes that are better than those achieved in other studies. The clinical models appear to work for the populations they serve. The Peer Social Support group worked for low-income persons with a limited social network. The Comprehensive Clinic Care group worked for urban, working persons with stable networks. Both treatment approaches are consistent with WHO (2014) recommendations that health care providers deliver ART services in a flexible manner depending on the communities they serve and according to the needs of the people with HIV infections. Unge et al. (2010) recommendations for more community-based treatment methods to address the challenges of the increasing number of individuals on life-long ART.

\section{Recommendations}

Considering the scarcity of personnel and funds in Kenya to address the rising demands for treatment for HIV infections and their precursors, it is important to scale up program(s) that work in order to channel public health efforts in a cost effective manner. Since much of Kenya's population resides in the rural areas, expanding the demonstrably effective Peer Social Support clinical model would appear to be a cost effective community-based public health strategy. But as nurses manage these clinics, there is a need to have more nurses trained and equipped to provide this care.

This study also highlighted the sporadic use of CD4 counts in both clinical models. Until recently, CD4 counts were used both to place people on ART and to assess the effectiveness of treatment. The lack of consistent records for CD4 counts was attributed to the cost for these tests. The current best practice for monitoring the effectiveness of ART is to assess the viral load. This test is even more expensive. For Kenya and other countries to deal effectively with the progression of this disease due to large numbers of individuals infected, either more funds must be directed at both urban and rural clinics to lower the costs to individuals for these tests or other accessible and 
affordable indexes to monitor progress for HIV patients must be found. Finally policy makers and programme implementers in low income resource setting should tailor their community based care services to respond to the needs of each social economic group they serve for people with HIV infection

\section{Acknowledgments}

We would like to express our sincere appreciation to the Decker School of Nursing in Binghamton University and Kasarani community based health center staff for support.

\section{References}

Bekker LG, Myer L, Orrell C, Lawn S, Wood R (2006) Rapid scale-up of a community-based HIV treatment service. South African Medical Journal 96(4): 315-322.

Campbell DT, Stanley JC, Gage NL (1963) Experimental and quasi-experimental designs for research. Houghton Mifflin Boston.

Broder S (2010) the development of antiretroviral therapy and its impact on the HIV1/AIDS pandemic. Antiviral Research 85(1): 1-18.

Fox MP, Rosen S (2010) Patient retention in antiretroviral therapy programs up to three years on treatment in sub-Saharan Africa, 2007-2009: Systematic review. Tropical Medicine \& International Health, 15(s1), 1-15

Ion A, Cai W, Elston D, Pullenayegum E, Smaill F, Smieja M (2011) A comparison of the MOS-HIV and SF-12v2 for measuring health-related quality of life of men and women living with HIV/AIDS. AIDS Research \& Therapy 8(5). DOI: 10.1186/1 742-6405-8-5.

Kabore I, Bloem J, Etheredge G, Obiero W, Wanless S, Doykos P et al. (2010) the effect of community-based support services on clinical efficacy and healthrelated quality of life in HIV/AIDS patients in resource-limited settings in subSaharan Africa. AIDS Patient Care and STDs 24(9): 581-594.

Kaplan JE, Hanson DL, Cohn DL, Karon J, Buskin S, Thompson M et al. (2003) When to begin highly active antiretroviral therapy? Evidence supporting initiation of therapy at CD4+ lymphocyte counts $<350$ cells $/ \mu \mathrm{L}$. Clinical Infectious Diseases 37(7): 951-958.

Lewden C, Chêne G, Morlat P, Raffi F, Dupon M, Dellamonica P et al. (2007) HIVinfected adults with a CD4 cell count greater than 500 cells $/ \mathrm{mm} 3$ on long-term combination antiretroviral therapy reach same mortality rates as the general population. Journal of Acquired Immune Deficiency Syndromes 46(1): 72-77.

Luo X, George ML, Kakouras I, Edwards CL, Pietrobon R, Richardson W, Hey L (2003) Reliability, validity, and responsiveness of the short form 12-item survey (SF-12) in patients with back pain. Spine 28(15): 1739-1745.

Nachega JB, Knowlton AR, Deluca A, Schoeman JH, Watkinson L, Efron A et al. (2006) Treatment supporter to improve adherence to antiretroviral therapy in HIV-infected South African adults: a qualitative study. Journal of Acquired Immune Deficiency Syndromes 43: S127-S133. 
Pop-Eleches C, Thirumurthy H, Habyarimana J, Graff Zivin J, Goldstein M, de Walque D et al. (2011) Mobile phone technologies improve adherence to antiretroviral treatment in resource-limited settings: A randomized controlled trial of text message reminders. AIDS 25: 825-834.

Rosen S, Fox MP (2011) Retention in HIV care between testing and treatment in subSaharan Africa: A systematic review. PLoS Medicine 8(7).

Unge C, Södergård B, Marrone G, Thorson A, Lukhwaro A, Carter J, Ekström AM (2010) Long-term adherence to antiretroviral treatment and program drop-out in a high-risk urban setting in sub-Saharan Africa: A prospective cohort study. PLoS One 5(10).

Visiwanatha H, Anderson R, Thomas III J (2005) Nature and correlates of SF-12 physical and mental quality of life components among low-income HIV adults using an HIVM service center. Quality of Life Research 14: 935-944.

WHO-World Health Organization (2006) WHO case definitions of HIV for surveillance and revised clinical staging and immunological classification of HIV-related disease in adults and children. World Health Organization.

WHO-World Health Organization (2014) March 2014 supplement to the 2013 consolidated guidelines on the use of antiretroviral drugs for treating and preventing HIV infection: recommendations for a public health approach. (Publication on HIV) Retrieved from http://goo.gl/jZ3que. 